\title{
New Variants of Defect Correction for Boundary VAlue Problems in Ordinary Differential Equations
}

\author{
Winfried AUZINGER \\ OTHMAR KOCH \\ Ewa B. WeinMüLLeR
}

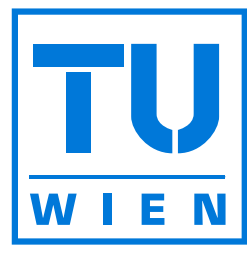

TECHNISCHE

UNIVERSITÄT

WIEN

VienNA

UNIVERSITY OF

TECHNOLOGY

Institute for Applied Mathematics

AND Numerical ANalysis 


\title{
New Variants of Defect Correction for Boundary Value Problems in Ordinary Differential Equations
}

\author{
Winfried Auzinger, Othmar Koch, and Ewa Weinmüller
}

\begin{abstract}
In this paper we discuss new variants of the acceleration technique known as Iterated Defect Correction (IDeC) for the numerical solution of boundary value problems in ordinary differential equations. A first approximation, computed by the backward Euler scheme, is iteratively improved to obtain a high order solution. Typically, the maximal attainable accuracy is limited by the smoothness of the exact solution and by technical details of the procedure. We propose a new version of the IDeC algorithm with maximal achievable order higher than in the classical setting. Moreover, our procedure can be shown to be convergent on arbitrary grids, while the classical IDeC iteration requires piecewise equidistant grids. Finally, the performance of this new algorithm for singular boundary value problems is discussed.
\end{abstract}

\section{Introduction}

We consider nonlinear boundary value problems for ordinary differential equations,

$$
\begin{aligned}
& z^{\prime}(t)=F(t, z(t)), \quad t \in[a, b], \\
& B_{a} z(a)+B_{b} z(b)=\beta,
\end{aligned}
$$

where $F(t, z)$ is a nonlinear $n$-dimensional function which is Lipschitz continuous w.r.t. $z$, and $B_{a}, B_{b} \in \mathbb{R}^{n \times n}$ are defined in such a way that the problem (1.1) is well-posed, cf. [1]. We assume that the solution $z(t) \in \mathbb{R}^{n}$ is sufficiently smooth and restrict our attention to linear boundary conditions (1.1b).

The solution method under consideration is based on the acceleration technique known as Iterated Defect Correction, introduced by Frank [4] and Stetter [10]. Here, in each step of the iteration a so-called "neighboring problem" to the original problem (1.1) is constructed. Both problems differ only in the defect function occurring in the right-hand side of the neighboring problem. This defect is the residual of the current numerical approximation w.r.t. (1.1a), based on a piecewise polynomial interpolant. The difference of the numerical solutions of the neighboring and original problems yields an error estimate which is used to improve the order

2000 Mathematics Subject Classification. Primary 65L05.

Key words and phrases. Iterated defect correction, boundary value problems, ordinary differential equations.

(C)0000 (copyright holder) 
of the approximation. The maximal attainable convergence order is limited by the degree of interpolation and by the smoothness of the exact solution $z(t)$. We stress that in the original version of the IDeC method a pointwise evaluation of the defect function is used.

In this paper, we propose an alternative variant of the IDeC procedure. Instead of the pointwise evaluation of the defect function we use its locally integrated values. Advantageously, the final numerical solution of the IDeC iteration shows a convergence order which is higher than in the classical procedure. Moreover, this high order approximation can be obtained on an arbitrary grid, whereas the original version requires the grid to be piecewise equidistant.

The idea of using a locally interpolated defect can also be successfully applied to obtain an asymptotically correct a posteriori estimate for the global error of collocation schemes, see [2] for the application of this idea to both regular and singular boundary value problems.

\section{Convergence Results}

Denote by $\eta_{\Delta}^{[0]}$ the basic numerical solution of (1.1) computed with the backward Euler method on a grid $\Delta:=\left\{t_{i, j}: i=0, \ldots, N-1, j=0, \ldots, m\right\}$. Note that we assume $t_{i-1, m}=t_{i, 0}$ and use this redundant indexing for notational convenience. Moreover, we only consider grids which satisfy $t_{i, j}=t_{i, 0}+\rho_{j}\left(t_{i, m}-t_{i, 0}\right), 0=\rho_{0}<$ $\rho_{1}<\cdots<\rho_{m}=1$. In addition, we set $\delta_{i, j}:=t_{i, j}-t_{i, j-1}$, and $\mathbf{h}:=\max _{i, j} \delta_{i, j}$. The norm on the space of grid vectors $u_{\Delta}$ defined on $\Delta$ is

$$
\left\|u_{\Delta}\right\|_{\Delta}:=\max _{i=0, \ldots N-1, j=0, \ldots, m-1}\left|u_{i, j}\right| .
$$

For a function $g$ defined on the interval $[a, b]$ the pointwise restriction onto the space of grid vectors is given as

$$
R_{\Delta}(g):=\left(g\left(t_{0,0}\right), \ldots, g\left(t_{N-1, m}\right)\right) .
$$

The basic approximation is the solution of

$$
\frac{\eta_{i, j}^{[0]}-\eta_{i, j-1}^{[0]}}{\delta_{i, j}}=F\left(t_{i, j}, \eta_{i, j}^{[0]}\right), \quad i=0, \ldots, N-1, j=1, \ldots, m,
$$

with boundary conditions (1.1b). Now we successively improve this numerical approximation in the following way: Let

$$
\eta_{\Delta}^{[\nu+1]}:=\eta_{\Delta}^{[0]}-\left(\pi_{\Delta}^{[\nu]}-\eta_{\Delta}^{[\nu]}\right), \quad \nu=0, \ldots, m-1,
$$

where $\pi_{\Delta}^{[\nu]}$ is the solution of the modified backward Euler scheme ${ }^{1}$

$$
\frac{\pi_{i, j}^{[\nu]}-\pi_{i, j-1}^{[\nu]}}{\delta_{i, j}}=F\left(t_{i, j}, \pi_{i, j}^{[\nu]}\right)+d_{i, j}^{[\nu]},
$$

with the same boundary conditions (1.1b). Consequently, the $\eta_{\Delta}^{[\nu+1]}$ also satisfy (1.1b). The defect term in (2.3) is defined as

$$
d_{i, j}^{[\nu]}:=\frac{\eta_{i, j}^{[\nu]}-\eta_{i, j-1}^{[\nu]}}{\delta_{i, j}}-\sum_{k=0}^{m} \alpha_{j, k} F\left(t_{i, k}, \eta_{i, k}^{[\nu]}\right),
$$

\footnotetext{
${ }^{1}$ Unless otherwise stated, we assume $i=0, \ldots, N-1, j=1, \ldots, m$.
} 
where the coefficients $\alpha_{j, k}$ are chosen as the unique weights of polynomial quadrature rules of precision $m+1$ with abscissae $\rho_{0}, \ldots, \rho_{m}$ on the interval $[0,1]$.

For this procedure, the following convergence result holds:

THEOREM 2.1. If the solution $z$ of (1.1) is sufficiently smooth, $z \in C^{m+2}[a, b]$, then

$$
\begin{aligned}
& \left\|\eta_{\Delta}^{[\nu]}-R_{\Delta}(z)\right\|_{\Delta}=O\left(\mathbf{h}^{\nu+1}\right), \quad \nu=0, \ldots, m, \\
& \left\|\bar{\partial} \eta_{\Delta}^{[\nu]}-\bar{\partial} R_{\Delta}(z)\right\|_{\Delta}=O\left(\mathbf{h}^{\nu+1}\right), \quad \nu=0, \ldots, m, \\
& \left\|q^{[\nu]}-z\right\|_{\infty}=O\left(\mathbf{h}^{\nu+1}\right), \quad \nu=0, \ldots, m, \\
& \left\|q^{[\nu]^{\prime}}-z^{\prime}\right\|_{\infty}=O\left(\mathbf{h}^{\nu+1}\right), \quad \nu=0, \ldots, m-1 .
\end{aligned}
$$

Here, $q^{[\nu]}(t)=q_{i}^{[\nu]}(t), t \in\left[t_{i, 0}, t_{i, m}\right], i=0, \ldots, N-1$, is a continuous, piecewise polynomial function of degree $\leq m$ which interpolates the values of $\eta_{\Delta}^{[\nu]}$ at the points $t_{i, j}, i=0, \ldots, N-1, j=0, \ldots, m . \bar{\partial}$ denotes the difference quotient

$$
\bar{\partial} \eta_{\Delta}^{[\nu]}=\frac{\eta_{i, j}^{[\nu]}-\eta_{i, j-1}^{[\nu]}}{\delta_{i, j}}
$$

ProOF. We use induction over $\nu$. Let us introduce the auxiliary quantities

$$
\varepsilon_{\Delta}^{[\nu]}:=\eta_{\Delta}^{[\nu]}-R_{\Delta}(z), \quad \xi_{\Delta}^{[\nu]}:=\pi_{\Delta}^{[\nu]}-\eta_{\Delta}^{[\nu]}, \quad \nu=0, \ldots, m
$$

Note that these grid vectors satisfy homogeneous boundary conditions ${ }^{2}$, and that

$$
\varepsilon_{\Delta}^{[\nu]}=\varepsilon_{\Delta}^{[0]}-\xi_{\Delta}^{[\nu-1]}
$$

holds. Furthermore we define

$$
\phi^{[\nu]}(t):=F(t, z(t))-F\left(t, q^{[\nu]}(t)\right), \quad t \in[a, b] .
$$

$\underline{\nu=0}$ : It is clear that (2.5) holds, due to the fact that the backward Euler method has convergence order 1 , the function $F$ satisfies a Lipschitz condition, and from standard results for polynomial interpolation, see [2] or [5]. We also note that $\varepsilon_{\Delta}^{[0]}$ satisfies

$$
\frac{\varepsilon_{i, j}^{[0]}-\varepsilon_{i, j-1}^{[0]}}{\delta_{i, j}}=F\left(t_{i, j}, \eta_{i, j}^{[0]}\right)-\sum_{k=0}^{m} \alpha_{j, k} F\left(t_{i, k}, z\left(t_{i, k}\right)\right)+O\left(\mathbf{h}^{m+1}\right),
$$

where the $O\left(\mathbf{h}^{m+1}\right)$ - term is the truncation error of the quadrature rule defined by the $\alpha_{j, k}$ applied to $z^{\prime} \in C^{m+1}[a, b]$.

$\nu-1 \rightarrow \nu$ : We use (2.3) and (2.4) to write

$$
\begin{aligned}
\frac{\xi_{i, j}^{[\nu-1]}-\xi_{i, j-1}^{[\nu-1]}}{\delta_{i, j}} & =F\left(t_{i, j}, \pi_{i, j}^{[\nu-1]}\right)+d_{i, j}^{[\nu-1]}-\frac{\eta_{i, j}^{[\nu-1]}-\eta_{i, j-1}^{[\nu-1]}}{\delta_{i, j}} \\
& =F\left(t_{i, j}, \pi_{i, j}^{[\nu-1]}\right)-\sum_{k=0}^{m} \alpha_{j, k} F\left(t_{i, k}, \eta_{i, k}^{[\nu-1]}\right) .
\end{aligned}
$$

\footnotetext{
${ }^{2}$ For simplicity, we refrain from stating these conditions explicitly.
} 
Furthermore, (2.7)-(2.10) yield

$$
\begin{array}{ll}
\frac{\varepsilon_{i, j}^{[\nu]}-\varepsilon_{i, j-1}^{[\nu]}}{\delta_{i, j}}= & F\left(t_{i, j}, \eta_{i, j}^{[0]}\right)-F\left(t_{i, j}, z\left(t_{i, j}\right)\right)-\left(F\left(t_{i, j}, \pi_{i, j}^{[\nu-1]}\right)-F\left(t_{i, j}, \eta_{i, j}^{[\nu-1]}\right)\right) \\
& +\phi^{[\nu-1]}\left(t_{i, j}\right)-\sum_{k=0}^{m} \alpha_{j, k} \phi^{[\nu-1]}\left(t_{i, k}\right)+O\left(\mathbf{h}^{m+1}\right) .
\end{array}
$$

To estimate $\left\|\varepsilon_{\Delta}^{[\nu]}\right\|_{\Delta}$ we consider the terms on the right-hand side of (2.11). Clearly, for the quadrature coefficients $\alpha_{j, k}$ we have $\sum_{k=0}^{m} \alpha_{j, k}=0$ for all $j$, and thus,

$$
\left|\phi^{[\nu-1]}\left(t_{i, j}\right)-\sum_{k=0}^{m} \alpha_{j, k} \phi^{[\nu-1]}\left(t_{i, k}\right)\right| \leq C \mathbf{h} \max _{t_{i, 0} \leq t \leq t_{i, m}}\left|\phi^{[\nu-1]^{\prime}}(t)\right|,
$$

where the total derivative $\phi^{[\nu-1]^{\prime}}$ satisfies

$$
\begin{aligned}
\phi^{[\nu-1]^{\prime}}(t)= & \frac{\partial}{\partial t} F(t, z(t))-\frac{\partial}{\partial t} F\left(t, q^{[\nu-1]}(t)\right) \\
& +\frac{\partial}{\partial z} F(t, z(t)) z^{\prime}(t)-\frac{\partial}{\partial z} F\left(t, q^{[\nu-1]}(t)\right) q^{[\nu-1]^{\prime}}(t)=O\left(\mathbf{h}^{\nu}\right)
\end{aligned}
$$

due to the inductive assumption that the assertion of the theorem holds for $\nu-1$.

Using Taylor's Theorem we further conclude

$$
\begin{aligned}
& F\left(t_{i, j}, \eta_{i, j}^{[0]}\right)-F\left(t_{i, j}, z\left(t_{i, j}\right)\right)=\int_{0}^{1} D F\left(t_{i, j}, z\left(t_{i, j}\right)+\tau \varepsilon_{i, j}^{[0]}\right) d \tau \cdot \varepsilon_{i, j}^{[0]} \\
& F\left(t_{i, j}, \pi_{i, j}^{[\nu-1]}\right)-F\left(t_{i, j}, \eta_{i, j}^{[\nu-1]}\right)=\int_{0}^{1} D F\left(t_{i, j}, \eta_{i, j}^{[\nu-1]}+\tau \xi_{i, j}^{[\nu-1]}\right) d \tau \cdot \xi_{i, j}^{[\nu-1]}
\end{aligned}
$$

To estimate the difference of the integrands in (2.13) and (2.14), we first derive a bound for the defect $d_{\Delta}^{[\nu-1]}$. To this end we use an auxiliary function $Q(t)$ which is the collocating polynomial of degree $m+1$ with collocation nodes $t_{i, 0}, \ldots, t_{i, m}$ for (1.1). Now, proceeding from (2.4), we write

$$
\begin{aligned}
d_{i, j}^{[\nu-1]}= & \frac{q^{[\nu-1]}\left(t_{i, j}\right)-q^{[\nu-1]}\left(t_{i, j-1}\right)}{\delta_{i, j}}-\frac{z\left(t_{i, j}\right)-z\left(t_{i, j-1}\right)}{\delta_{i, j}} \\
& +\frac{z\left(t_{i, j}\right)-z\left(t_{i, j-1}\right)}{\delta_{i, j}}-\frac{Q\left(t_{i, j}\right)-Q\left(t_{i, j-1}\right)}{\delta_{i, j}} \\
& +\frac{Q\left(t_{i, j}\right)-Q\left(t_{i, j-1}\right)}{\delta_{i, j}}-\sum_{k=0}^{m} \alpha_{j, k} F\left(t_{i, k}, Q\left(t_{i, k}\right)\right) \\
& +\sum_{k=0}^{m} \alpha_{j, k}\left(F\left(t_{i, k}, Q\left(t_{i, k}\right)\right)-F\left(t_{i, k}, q^{[\nu-1]}\left(t_{i, k}\right)\right)\right) .
\end{aligned}
$$

The right-hand side of (2.15) is $O\left(\mathbf{h}^{\nu}\right)$ due to the inductive assumption (2.5b) for $\nu-1$, and the same can be shown for (2.18) using the triangle inequality with the auxiliary terms $F\left(t_{i, k}, z\left(t_{i, k}\right)\right)$. For $(2.17)$ we have

$$
\begin{aligned}
\frac{Q\left(t_{i, j}\right)-Q\left(t_{i, j-1}\right)}{\delta_{i, j}} & =\frac{1}{\delta_{i, j}} \int_{t_{i, j-1}}^{t_{i, j}} Q^{\prime}(\tau) d \tau=\sum_{k=0}^{m} \alpha_{j, k} Q^{\prime}\left(t_{i, k}\right)+O\left(\mathbf{h}^{m+1}\right) \\
& =\sum_{k=0}^{m} \alpha_{j, k} F\left(t_{i, k}, Q\left(t_{i, k}\right)\right)+O\left(\mathbf{h}^{m+1}\right) .
\end{aligned}
$$


Finally we prove that (2.16) is $O\left(\mathbf{h}^{m+1}\right)$ using Taylor expansion at $t_{i, j}$ and making use of the fact that the derivatives of the collocating function $Q$ satisfy

$$
\left\|Q^{(l)}-z^{(l)}\right\|_{\infty}=O\left(\mathbf{h}^{m+2-l}\right), \quad l=1, \ldots, m+1,
$$

if $z \in C^{m+2}[a, b]$, see for example [1]. Thus,

$$
\left|d_{i, j}^{[\nu-1]}\right|=O\left(\mathbf{h}^{\nu}\right),
$$

and with (2.1) and (2.3) we conclude

$$
\left|\eta_{i, j}^{[0]}-\pi_{i, j}^{[\nu-1]}\right|=O\left(\mathbf{h}^{\nu}\right)
$$

due to the stability of the backward Euler scheme, see [1]. For the integrands in (2.13) and (2.14) this implies

$$
\left(z\left(t_{i, j}\right)-\eta_{i, j}^{[\nu-1]}\right)(1-\tau)+\left(\eta_{i, j}^{[0]}-\pi_{i, j}^{[\nu-1]}\right) \tau=O\left(\mathbf{h}^{\nu}\right),
$$

and using the Lipschitz condition which $D F$ satisfies we obtain

$$
\begin{aligned}
F\left(t_{i, j}, \eta_{i, j}^{[0]}\right)- & F\left(t_{i, j}, z\left(t_{i, j}\right)\right)-\left(F\left(t_{i, j}, \pi_{i, j}^{[\nu-1]}\right)-F\left(t_{i, j}, \eta_{i, j}^{[\nu-1]}\right)\right) \\
= & A\left(t_{i, j}\right) \varepsilon_{i, j}^{[\nu]}+B\left(t_{i, j}\right)\left(\pi_{i, j}^{[\nu-1]}-\eta_{i, j}^{[\nu-1]}\right) \\
& =A\left(t_{i, j}\right) \varepsilon_{i, j}^{[\nu]}+O\left(\mathbf{h}^{\nu+1}\right) .
\end{aligned}
$$

Consequently, (2.11) may be written as a linear difference equation of the backward Euler type, with an inhomogeneous part which is $O\left(\mathbf{h}^{\nu+1}\right)$. The stability of the scheme now yields $(2.5 \mathrm{a})$ while $(2.5 \mathrm{~b})$ follows from $(2.11)$. Let us denote by $q^{*}(t)$ the piecewise polynomial function of degree $\leq m$, interpolating the values of $z(t)$ in the abscissae $t_{i, 0}, \ldots, t_{i, m}$. Then (2.5c) follows from

$$
\left\|q^{[\nu]}-z\right\|_{\infty} \leq\left\|q^{[\nu]}-q^{*}\right\|_{\infty}+\left\|q^{*}-z\right\|_{\infty}
$$

and standard results for polynomial interpolation, see [5].

To prove $(2.5 \mathrm{~d})$ we require the following result:

For abscissae $\rho_{0}, \ldots, \rho_{m} \in[0,1]$, every polynomial $P$ of degree $\leq m$ and $\bar{t} \in$ $[0,1]$, there are unique coefficients $\beta_{1}(\bar{t}), \ldots, \beta_{m}(\bar{t})$, such that

$$
P^{\prime}(\bar{t})=\sum_{k=1}^{m} \beta_{k}(\bar{t}) \frac{P\left(\rho_{k}\right)-P\left(\rho_{k-1}\right)}{\rho_{k}-\rho_{k-1}} .
$$

The $\beta_{k}(\bar{t})$ are uniformly bounded in $\bar{t}$ and in $\mathbf{h}$, if we consider the relation corresponding to (2.19) for the nodes $t_{i, 0}, \ldots, t_{i, m} \in \Delta$. These facts can easily be concluded from the representation

$$
\beta_{k}(\bar{t})=-\left(\rho_{k}-\rho_{k-1}\right) \sum_{l=0}^{k-1} L_{l}^{\prime}(\bar{t}),
$$

where $L_{l}(t)$ are the Lagrange polynomials associated with the abscissae $\rho_{0}, \ldots, \rho_{m}$.

Using this latter result and the inductive assumption (2.5b) we have

$$
\left(q^{[\nu]^{\prime}}-q^{*^{\prime}}\right)(t)=\sum_{k=1}^{m} \beta_{k}(t) \frac{\varepsilon_{i, k}^{[\nu]}-\varepsilon_{i, k-1}^{[\nu]}}{\delta_{i, k}}=O\left(\mathbf{h}^{\nu+1}\right) .
$$

Now, for $\nu<m,(2.5 \mathrm{~d})$ follows from

$$
\left\|q^{[\nu]^{\prime}}-z^{\prime}\right\|_{\infty} \leq\left\|q^{[\nu]^{\prime}}-q^{*^{\prime}}\right\|_{\infty}+\left\|q^{*^{\prime}}-z^{\prime}\right\|_{\infty},
$$


due to $(2.20)$, and due to $\left\|q^{*^{\prime}}-z^{\prime}\right\|_{\infty}=O\left(\mathbf{h}^{m}\right)$, see [5]. From this, we finally note that the estimate $(2.5 \mathrm{~d})$ is indeed not valid for $\nu=m$.

REMARK 2.2. We stress that the arguments used in the proof of Theorem 2.1 are not applicable for the classical IDeC version with pointwise defect, where piecewise equidistant grids would be necessary.

Finally, we would like to comment on an interesting property of the collocating function $Q(t)=Q_{i}(t), t \in\left[t_{i, 0}, t_{i, m}\right], i=0, \ldots, N-1$, introduced in the proof of Theorem 2.1. Since $Q_{i}^{\prime}$ is a polynomial of degree $\leq m$, we have

$$
\begin{aligned}
\frac{Q_{i}\left(t_{i, j}\right)-Q_{i}\left(t_{i, j-1}\right)}{\delta_{i, j}} & =\frac{1}{\delta_{i, j}} \int_{t_{i, j-1}}^{t_{i, j}} Q_{i}^{\prime}(\tau) d \tau \\
& =\sum_{k=0}^{m} \alpha_{j, k} Q_{i}^{\prime}\left(t_{i, k}\right)=\sum_{k=0}^{m} \alpha_{j, k} F\left(t_{i, k}, Q_{i}\left(t_{i, k}\right)\right) .
\end{aligned}
$$

This means that the defect (2.4) evaluated for $Q$ vanishes at all grid points. Consequently, the fixed point $Q_{\Delta}$ of the IDeC iteration satisfies

$$
Q_{i, j}=Q\left(t_{i, j}\right)
$$

Conversely, the collocating function $Q$ can be retrieved from this fixed point $Q_{\Delta}$ using Hermite interpolation,

$$
\begin{aligned}
& Q\left(t_{i, j}\right)=Q_{i, j}, \quad i=0, \ldots, N-1, j=0, \ldots, m \\
& Q^{\prime}\left(t_{i, 0}\right)=F\left(t_{i, 0}, Q_{i, 0}\right), \quad i=0, \ldots, N-1 .
\end{aligned}
$$

For this function,

$$
\begin{aligned}
& \|Q-z\|_{\infty}=O\left(\mathbf{h}^{m+1}\right), \\
& \left\|Q^{\prime}-z^{\prime}\right\|_{\infty}=O\left(\mathbf{h}^{m+1}\right),
\end{aligned}
$$

holds. This enables us to improve the uniform approximation of $z^{\prime}$, cf. (2.5d), if necessary.

\section{Singular Problems}

Many standard numerical methods do not work efficiently when applied to singular boundary value problems, see for example [7], because order reductions negatively affect their performance. Concerning defect correction, there has been some promising experience, see [2] and [9], which suggests to apply the method described above to singular problems. Unfortunately, we observe an order reduction for the global error down to order 2, in general. As an example, consider the Emden differential equation,

$$
\begin{aligned}
& z^{\prime}(t)=\frac{1}{t}\left(\begin{array}{cc}
0 & 1 \\
0 & -1
\end{array}\right) z(t)-\left(\begin{array}{c}
0 \\
t z_{1}^{5}(t)
\end{array}\right), \quad t \in(0,1] \\
& \left(\begin{array}{ll}
0 & 0 \\
0 & 1
\end{array}\right) z(0)+\left(\begin{array}{ll}
1 & 0 \\
0 & 0
\end{array}\right) z(1)=\left(\begin{array}{c}
\frac{\sqrt{3}}{2} \\
0
\end{array}\right) .
\end{aligned}
$$

The exact solution of this problems is given by

$$
z(t)=\left(z_{1}(t), z_{2}(t)\right)^{T}=\left(\frac{1}{\sqrt{1+t^{2} / 3}},-\frac{t^{2}}{3 \sqrt{\left(1+t^{2} / 3\right)^{3}}}\right)^{T} .
$$


Table 1 displays the exact global errors and empirical convergence rates for the successive iterates in the above IDeC variant, for the choice $m=4$ and $\rho_{j}=j / 4$. All computations were carried out in MATLAB, using IEEE double precision arithmetic with machine accuracy eps $\approx 1.11 \cdot 10^{-16}$. The results show no convergence order higher than 2 .

TABLE 1. Convergence orders of IDeC iterates for (3.1).

\begin{tabular}{|r||c|c|c|c|c|c|}
\hline \multicolumn{1}{|c|}{$N$} & err $_{0}$ & $\operatorname{ord}_{0}$ & err $_{1}$ & ord $_{1}$ & err $_{2}$ & ord $_{2}$ \\
\hline 16 & $3.46 \mathrm{e}-03$ & & $1.66 \mathrm{e}-05$ & & $2.13 \mathrm{e}-06$ & \\
32 & $1.73 \mathrm{e}-03$ & 1.00 & $4.11 \mathrm{e}-06$ & 2.01 & $4.59 \mathrm{e}-07$ & 2.22 \\
64 & $8.68 \mathrm{e}-04$ & 1.00 & $1.02 \mathrm{e}-06$ & 2.01 & $1.05 \mathrm{e}-07$ & 2.12 \\
128 & $4.34 \mathrm{e}-04$ & 1.00 & $2.54 \mathrm{e}-07$ & 2.01 & $2.52 \mathrm{e}-08$ & 2.06 \\
256 & $2.17 \mathrm{e}-04$ & 1.00 & $6.35 \mathrm{e}-08$ & 2.00 & $6.18 \mathrm{e}-09$ & 2.03 \\
\hline
\end{tabular}

The situation changes however, when we modify our procedure by applying a quadrature rule of a lower order, namely of precision $m$, where the solution values at $t_{i, 1}, \ldots, t_{i, m}$ are used in the definition of the defect (2.4). In this case, the highest attainable orders in the estimates (2.5) are only $O\left(\mathbf{h}^{m}\right)$. This result can be shown analytically for regular problems using analogous arguments as in the proof of Theorem 2.1.

It turns out that this method yields the full convergence order $m$ after $m-1$ iteration steps for problem (3.1). In Table 2 the results for $m=4$ are given (we leave out the results for the basic scheme, which in $[6]$ was proven to have order 1 ).

TABLE 2. Convergence orders of lower order IDeC for (3.1).

\begin{tabular}{|r||c|c|c|c|c|c|c|c|}
\hline \multicolumn{1}{|c||}{$N$} & $\mathrm{err}_{1}$ & ord $_{1}$ & $\mathrm{err}_{2}$ & ord $_{2}$ & $\mathrm{err}_{3}$ & ord $_{3}$ & err $_{4}$ & ord $_{4}$ \\
\hline 4 & $2.59 \mathrm{e}-04$ & & $3.77 \mathrm{e}-05$ & & $7.85 \mathrm{e}-06$ & & $6.99 \mathrm{e}-06$ & \\
8 & $6.85 \mathrm{e}-05$ & 1.92 & $4.55 \mathrm{e}-06$ & 3.05 & $4.76 \mathrm{e}-07$ & 4.04 & $4.33 \mathrm{e}-07$ & 4.01 \\
16 & $1.66 \mathrm{e}-05$ & 2.04 & $5.67 \mathrm{e}-07$ & 3.01 & $2.95 \mathrm{e}-08$ & 4.01 & $2.69 \mathrm{e}-08$ & 4.01 \\
32 & $4.11 \mathrm{e}-06$ & 2.01 & $7.03 \mathrm{e}-08$ & 3.01 & $1.83 \mathrm{e}-09$ & 4.01 & $1.68 \mathrm{e}-09$ & 4.00 \\
64 & $1.02 \mathrm{e}-06$ & 2.01 & $8.75 \mathrm{e}-09$ & 3.01 & $1.14 \mathrm{e}-10$ & 4.01 & $1.04 \mathrm{e}-10$ & 4.00 \\
\hline
\end{tabular}

This behavior is analogous to that for the classical IDeC iteration (with pointwise defect), see $[\mathbf{3}]$ and $[\mathbf{9}]$.

For some other singular problems, it is known that the classical IDeC variant shows order reductions. We consider the following example to demonstrate that our new lower order variant is not able to overcome this drawback:

$$
\begin{aligned}
& z^{\prime}(t)=\frac{1}{t}\left(\begin{array}{ll}
0 & 1 \\
1 & 0
\end{array}\right) z(t)+\left(\begin{array}{c}
0 \\
3 t \cos (t)-t^{2} \sin (t)
\end{array}\right), \quad t \in(0,1] \\
& \left(\begin{array}{ll}
0 & 1 \\
0 & 0
\end{array}\right) z(0)+\left(\begin{array}{ll}
0 & 0 \\
1 & 0
\end{array}\right) z(1)=\left(\begin{array}{c}
0 \\
\sin (1)
\end{array}\right) .
\end{aligned}
$$

The exact solution of $(3.2)$ reads

$$
z(t)=\left(t \sin (t), t \sin (t)+t^{2} \cos (t)\right)^{T}
$$

For $m=4$ the iteration has an order sequence $O(\mathbf{h}), O\left(\mathbf{h}^{2}\right), O\left(\mathbf{h}^{2}\right)$, etc., cf. Table 3 . The same behavior can be observed for the classical IDeC method, see [3] and [8]. 
TABle 3. Convergence orders of lower order IDeC for (3.2).

\begin{tabular}{|r||c|c|c|c|c|c|}
\hline \multicolumn{1}{|c||}{$N$} & $\operatorname{err}_{0}$ & $\operatorname{ord}_{0}$ & $\mathrm{err}_{1}$ & $\operatorname{ord}_{1}$ & $\mathrm{err}_{2}$ & $\operatorname{ord}_{2}$ \\
\hline 4 & $1.83 \mathrm{e}-02$ & & $6.84 \mathrm{e}-03$ & & $3.79 \mathrm{e}-03$ & \\
8 & $8.91 \mathrm{e}-03$ & 1.04 & $1.72 \mathrm{e}-03$ & 1.99 & $9.60 \mathrm{e}-04$ & 1.98 \\
16 & $4.48 \mathrm{e}-03$ & 0.99 & $4.31 \mathrm{e}-04$ & 2.00 & $2.40 \mathrm{e}-04$ & 2.00 \\
32 & $2.22 \mathrm{e}-03$ & 1.01 & $1.07 \mathrm{e}-04$ & 2.00 & $6.02 \mathrm{e}-05$ & 2.00 \\
64 & $1.10 \mathrm{e}-03$ & 1.00 & $2.69 \mathrm{e}-05$ & 2.00 & $1.50 \mathrm{e}-05$ & 2.00 \\
\hline
\end{tabular}

We thus observe that neither classical nor modified variants of IDeC work fully satisfactorily for general singular problems. Only problems which can be equivalently formulated as a well-posed initial value problem (this is the case for (3.1)) are in the scope of these methods, see [9]. Thus, concerning the application of IDeC methods to singular problems, some interesting questions remain unanswered.

Alternatively, collocation methods can be applied successfully. Here, locally integrated defects can also be used for the design of an asymptotically correct a posteriori estimate of the global error. See [2] for details of such a procedure.

\section{Acknowledgement}

We wish to thank Stefan Lammer for his implementation of the test routines.

\section{References}

1. U. Ascher, R.M.M. Mattheij, and R.D. Russell, Numerical solution of boundary value problems for ordinary differential equations, Prentice-Hall, Englewood Cliffs, NJ, 1988.

2. W. Auzinger, O. Koch, and E. Weinmüller, Efficient collocation schemes for singular boundary value problems, to appear in Numer. Algorithms. Also available as ANUM Preprint Nr. 5/01 at http://www.math.tuwien.ac.at/ inst115/preprints.htm.

3. _ Theory and solution techniques for singular boundary value problems for ordinary differential equations, Parallel Processing and Applied Mathematics (M. Paprzycki R. Wyrzykowski, J. Dongarra and J. Wasniewski, eds.), Springer Lecture Notes in Computer Science, vol. 2328, 2002, pp. 851-861. Also available as ANUM Preprint Nr. 6/01 at http://www . math.tuwien.ac.at/ inst115/preprints.htm,

4. R. Frank, The method of Iterated Defect Correction and its application to two-point boundary value problems, Part I, Numer. Math. 25 (1976), 409-419.

5. F. B. Hildebrand, Introduction to numerical analysis, $2^{\text {nd }}$ ed., McGraw-Hill, New York, 1974.

6. F.R. de Hoog and R. Weiss, Difference methods for boundary value problems with a singularity of the first kind, SIAM J. Numer. Anal. 13 (1976), 775-813.

7. _ The application of Runge-Kutta schemes to singular initial value problems, Math. Comp. 44 (1985), 93-103.

8. O. Koch and E. Weinmüller, Acceleration techniques for singular initial value problems, Problems in Modern Applied Mathematics (N. Mastorakis, ed.), WSES Press, 2000, pp. 6-11.

9. I I I J. Numer. Anal. 38 (2001), no. 6, 1784-1799.

10. H. J. Stetter, The defect correction principle and discretization methods, Numer. Math. 29 (1978), 425-443.

All: Institute for Applied Mathematics and Numerical Analysis, Vienna University of Technology, Austria

E-mail address: w.auzinger@tuwien.ac.at, othmar@fsmat.at, e.weinmueller@tuwien.ac.at 